\title{
Positivity violation for the lattice Landau gluon propagator
}

\author{
Attilio Cucchieri, ${ }^{1}$ Tereza Mendes, ${ }^{1}$ and Andre R. Taurines ${ }^{2}$ \\ ${ }^{1}$ Instituto de Física de São Carlos, Universidade de São Paulo, \\ C.P. 369, 13560-970, São Carlos, SP, Brazil \\ ${ }^{2}$ Instituto de Física Teórica, Universidade Estadual Paulista, \\ Rua Pamplona, 145, São Paulo, SP, Brazil
}

\begin{abstract}
We present explicit numerical evidence of reflection-positivity violation for the lattice Landau gluon propagator in three-dimensional pure $S U(2)$ gauge theory. We use data obtained at very large lattice volumes $\left(V=80^{3}, 140^{3}\right)$ and for three different lattice couplings in the scaling region $(\beta=$ $4.2,5.0,6.0)$. In particular, we observe a clear oscillatory pattern in the real-space propagator $C(t)$. We also verify that the (real-space) data show good scaling in the range $t \in[0,3] \mathrm{fm}$ and can be fitted using a Gribov-like form. The violation of positivity is in contradiction with a stable-particle interpretation of the associated field theory and may be viewed as a manifestation of confinement.
\end{abstract}

PACS numbers: 11.10.Cd, 11.15.Ha, 12.38.Aw, 14.70.Dj

\section{INTRODUCTION}

In recent years, there has been considerable interest in the possible violation of spectral positivity for QCD and in its relation to color confinement (for a review see [1]). Let us recall [2] that the reconstruction of a GårdingWightman relativistic quantum field theory from the corresponding Euclidean Green functions is possible only if they obey the Osterwalder-Schrader axioms [3]. In particular, the requirement of positive definiteness of the norm in Hilbert space is expressed in Euclidean space by the axiom of reflection positivity. For a generic 2-point function $\widetilde{D}(x-y)$, this axiom reads

$$
\int d^{4} x d^{4} y f^{*}\left(-x_{0}, \mathbf{x}\right) \widetilde{D}(x-y) f\left(y_{0}, \mathbf{y}\right) \geq 0,
$$

where $f\left(x_{0}, \mathbf{x}\right)$ is an arbitrary complex test function. The above condition implies the existence of a KällenLehmann representation for $\widetilde{D}(x-y)$, which is necessary for interpreting the fields in terms of stable particles. Thus, a violation of (11) implies that the Euclidean 2-point function cannot represent the correlations of a physical particle. This may be viewed as an indication of confinement [1].

The relation between reflection positivity and Euclidean correlation functions can be made explicit by considering the spectral representation [1, 4]

$$
D(p)=\int_{0}^{\infty} d m^{2} \frac{\rho\left(m^{2}\right)}{p^{2}+m^{2}}
$$

for the Euclidean propagator in momentum space. Then, the statement of reflection positivity is equivalent to a positive spectral density $\rho\left(\mathrm{m}^{2}\right)$. This implies that the temporal correlator at zero spatial momentum $D(t, \mathbf{p}=$ 0 ) can be written as

$$
C(t) \equiv D(t, 0)=\int_{0}^{\infty} d \omega \rho\left(\omega^{2}\right) e^{-\omega t} .
$$

We note that for general spatial momentum $\mathbf{p}$ one would have $\omega=\sqrt{\mathbf{p}^{2}+m^{2}}$. [In the particular case $\mathbf{p}=0$ con- sidered here, the decay behavior of $D(t, \mathbf{p})$ provides direct insight on mass-like properties associated with the fields.] Clearly, a positive density $\rho\left(\omega^{2}\right)$ implies that

$$
C(t)>0 \text {. }
$$

Notice that having $C(t)>0$ for all $t$ does not ensure the positivity of $\rho\left(\omega^{2}\right)$. On the other hand, finding $C(t)<0$ for some $t$ implies that $\rho\left(\omega^{2}\right)$ cannot be positive, suggesting confinement for the corresponding particle.

For the gluon, the Landau propagator is predicted to vanish at zero momentum [5, 6, 7, 8, 9, 10]. This implies that the real-space propagator $\widetilde{D}(x-y)$ is positive and negative in equal measure, i.e. reflection positivity is maximally violated [5, 6, []]. An infrared suppressed Landau gluon propagator has been obtained in several studies in momentum space [11, 12, 13, 14]. Numerical indications of a negative real-space lattice Landau gluon propagator have been presented in the $3 d S U(2)$ case [12], in the magnetic sector of the $4 d S U(2)$ case at finite temperature [13] and, recently, in the $4 d S U(3)$ case for one "exceptional" configuration [15]. In this work we verify this feature in detail (see Section I), using data obtained at very large lattice sizes for the $S U(2)$ case in three space-time dimensions 14]. At the same time, we try to fit the numerical data in real space (see Section IIII) by considering a sum of Gribov-like propagators [5, 7]. Let us recall that an excellent fit of the (momentum-space) propagator by a Gribov-like formula has been obtained for the equal-time three-dimensional transverse gluon propagator in $4 d S U(2)$ Coulomb gauge 16] and for the $3 d S U(2)$ Landau case [14], while in Ref. [4] the (real-space) transverse propagator has been fitted using a a Stingl-like formula in the $4 d S U(3)$ Landau case. Also, several fitting forms have been considered in Ref. 17] for the gluon propagator in momentum space.

\section{VIOLATION OF REFLECTION POSITIVITY}

An explicit (numerical) proof of violation of the condition (41) may be difficult if the correlation function 
$C(t)$ only becomes negative at relatively large values of $t$. In particular, this is the case if $C(t)$ is of the form $C(t)=e^{-\lambda t} f(t)$ and $f(t)$ is only negative for $t \gg 1 / \lambda$. In this case it is helpful to consider alternative quantities $G(t)$ that are positive if $\rho\left(\omega^{2}\right)$ is positive. [Consequently, finding $G(t)<0$ implies violation of positivity for $\rho\left(\omega^{2}\right)$.] For example, one can define [18] the function

$$
\begin{aligned}
G(t) & \equiv \frac{d^{2}}{d t^{2}} \ln C(t) \\
& =\frac{C(t) C^{\prime \prime}(t)-\left[C^{\prime}(t)\right]^{2}}{[C(t)]^{2}} .
\end{aligned}
$$

Using Eq. (3) we can write $G(t)$ as 1,4$]$

$$
G(t)=\left\langle(\omega-\langle\omega\rangle)^{2}\right\rangle,
$$

where the averages denoted by \langle\rangle are evaluated in the measure $d \omega \rho\left(\omega^{2}\right) e^{-\omega t}$. Clearly, if the density $\rho\left(\omega^{2}\right)$ is positive, so is $G(t)$. Let us note that for $C(t)=e^{-\lambda t} f(t)$ one gets $G(t) \equiv d^{2} \ln f(t) / d t^{2}$, namely we get rid of the exponential factor $e^{-\lambda t}$ and it should be easy to check numerically if $G(t)$ - or equivalently $f(t)$ - is negative. The quantity $G(t)$ could be of particular interest in a $4 d$ study, since in this case it is more difficult to obtain good data for large time separations. In the case of a Gribov-like momentum-space propagator [7]

$$
D(p)=p^{2} /\left(p^{4}+M^{4}\right),
$$

one obtains the real-space propagator $[5]$

$$
\begin{aligned}
C(t) & =\frac{1}{2 \pi} \int_{-\infty}^{\infty} d p D(p) e^{-i p t} \\
& =\frac{e^{-M t / \sqrt{2}}}{2 M} \cos \left(\frac{M t}{\sqrt{2}}+\frac{\pi}{4}\right) .
\end{aligned}
$$

Then, using Eq. (6), it is easy to check that

$$
G(t)=-M^{2}\left[2 \cos ^{2}\left(\frac{M t}{\sqrt{2}}+\frac{\pi}{4}\right)\right]^{-1},
$$

which is negative for all values of $t$.

Notice that if $C(t)$ is negative for some value of $t$ we cannot evaluate its logarithm in Eq. (5), while the expression in Eq. (6) is always well defined for $C(t) \neq 0$.

On the lattice, the real-space propagator can be evaluated using

$$
C(t)=\frac{1}{N} \sum_{k_{0}=0}^{N-1} e^{-2 \pi i k_{0} t / N} D\left(k_{0}, 0\right),
$$

where $N$ is the number of points per lattice side and $D(k)$ is the propagator in momentum space. If the lattice action satisfies reflection positivity [19], then we can write the spectral representation

$$
C(t)=\sum_{n} r_{n} e^{-E_{n} t}
$$
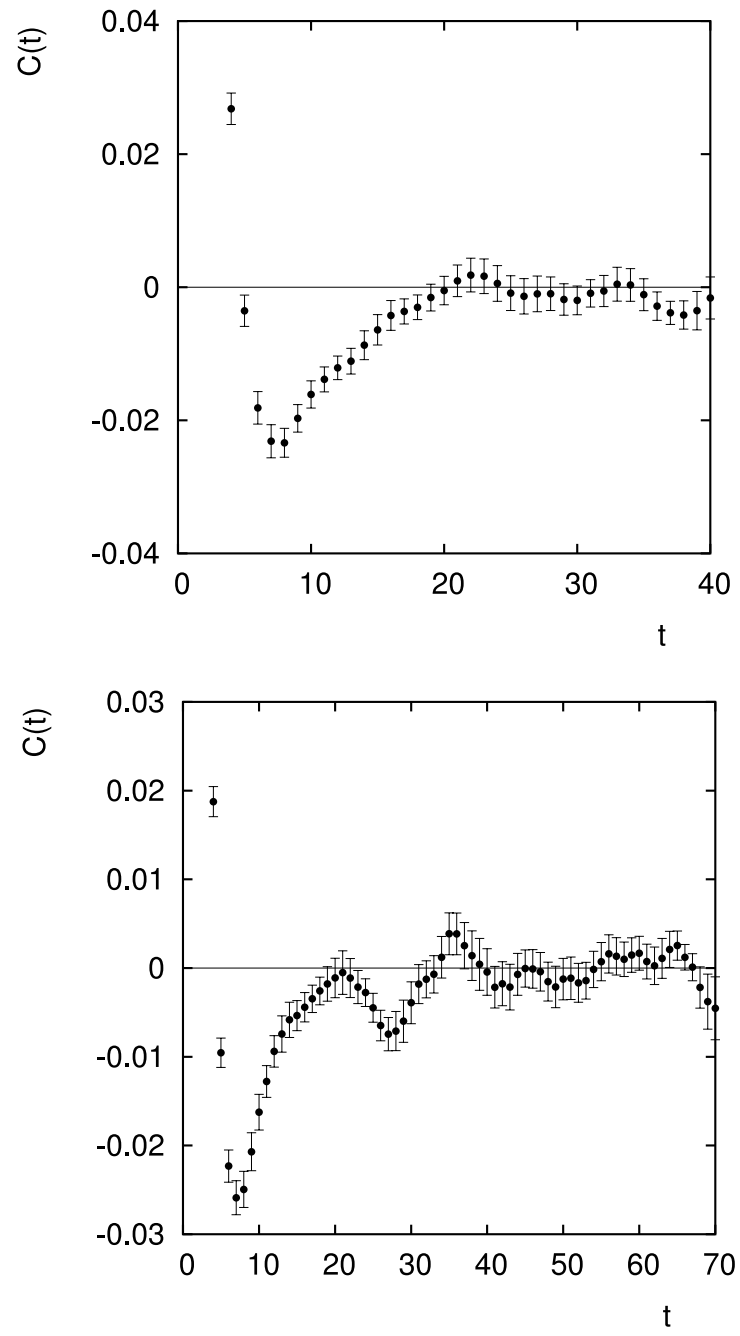

FIG. 1: Real-space propagator $C(t)$ as a function of $t$ for coupling $\beta=5.0$ and lattice volumes $V=80^{3}$ (above) and $V=140^{3}$ (below). Errors have been evaluated using bootstrap with 1000 samples. All quantities are in lattice units.

where $r_{n}$ are positive-definite constants. Clearly, this implies that $C(t)$ is non-negative for all values of $t$.

As in the continuum, we can consider $G(t)$ using Eq. (6) or, equivalently, the function $G(t)[C(t)]^{2}$. This quantity can be easily discretized on the lattice by

$$
G(t, a)=\frac{1}{a^{2}}\left[C(t) C(t+2 a)-C(t+a)^{2}\right],
$$

where $a$ is the lattice spacing. Indeed, in the continuum limit $a \rightarrow 0$, one obtains $G(t, a)=G(t) C(t)^{2}+\mathcal{O}\left(a^{3}\right)$. Furthermore, defining

$$
b_{n}=\sqrt{r_{n}} e^{-E_{n} t / 2}, \quad c_{n}=\sqrt{r_{n}} e^{-E_{n}(t+2 a) / 2},
$$

we can use the Schwartz inequality 18 to show that $G(t, a) \geq 0$ for all values of $t$ and $a$ if the $r_{n}$ 's are positive. Also, considering the effective gluon mass

$$
m(t)=-\log [C(t+a) / C(t)],
$$




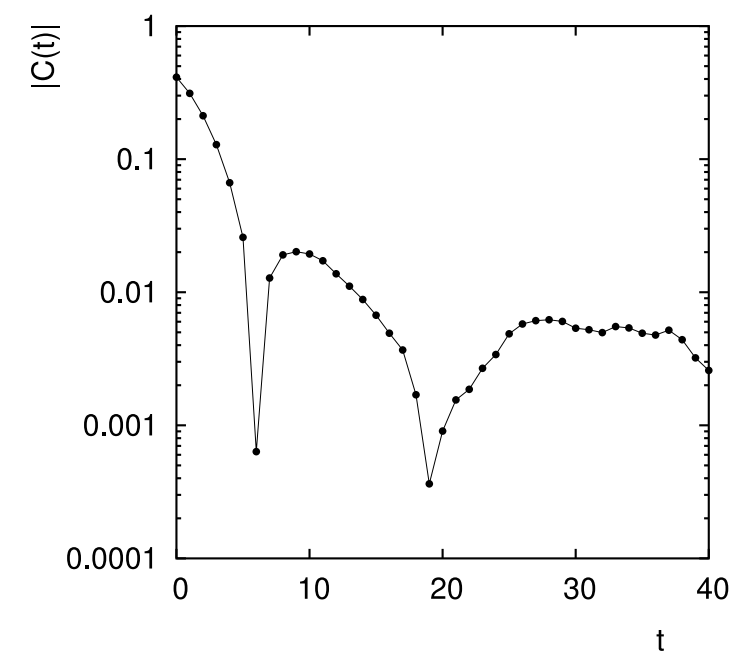

FIG. 2: Plot of $|C(t)|$ as a function of $t$ for lattice volume $V=80^{3}$ and coupling $\beta=6.0$. For clarity, errors are not shown. All quantities are in lattice units. The solid line is drawn to guide the eye.

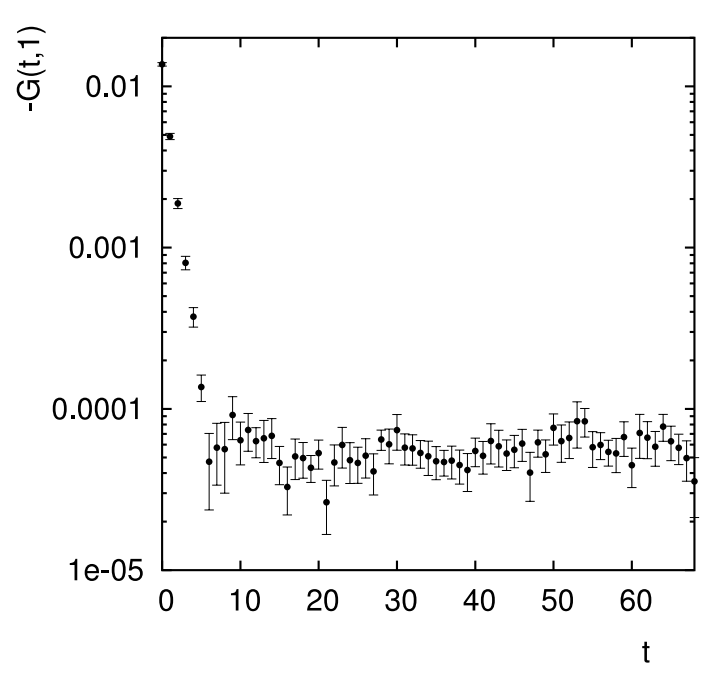

FIG. 3: Plot of $-G(t, 1)$ as a function of $t$ for lattice volume $V=140^{3}$ and coupling $\beta=4.2$. Errors have been evaluated using bootstrap with 1000 samples. All quantities are in lattice units.

one gets $e^{m(t)}-e^{m(t+a)}=G(t, a) a^{2} e^{m(t+a)} / C(t+a)^{2}$. Thus, if $G(t, a) \geq 0$ one obtains $m(t) \geq m(t+a)$, i.e. the effective mass should decrease when considering a larger time separation $t$. As discussed in [18], an increasing effective gluon mass has been obtained already in the first numerical studies of the gluon propagator 20], suggesting a violation of reflection positivity. Note, however, that Eq. (16) is ill-defined if $C(t)$ changes sign.

Here we use the $3 d S U(2)$ Landau-gauge data presented in 14] in order to check if the conditions $C(t)>0$ and $G(t, a)>0$ are violated for the gluon propagator. (The data have been analyzed using the bootstrap method with 1000 samples; we checked that results do not change when using 500 samples.) As explained in

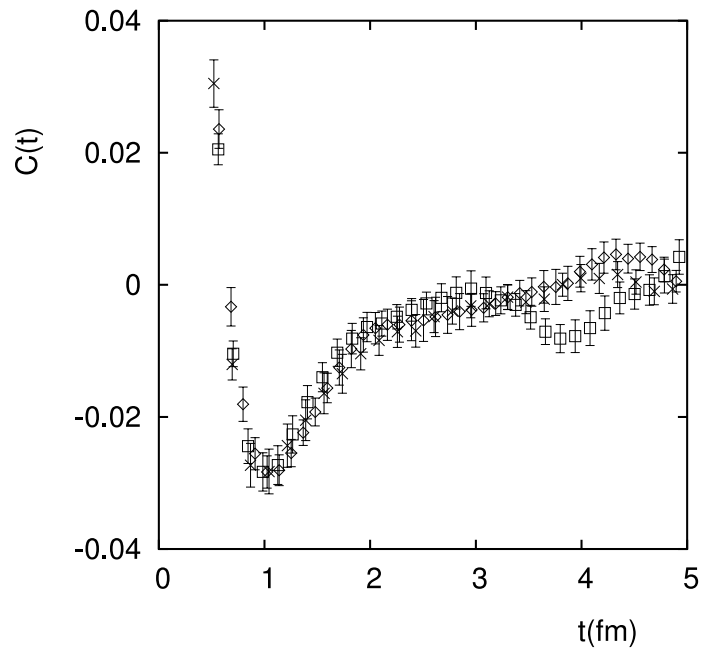

FIG. 4: Scaling for the real-space propagator $C(t)$ as a function of $t$ (in $\mathrm{fm}$ ) for lattice volume $V=140^{3}$ and couplings $\beta=4.2(\times), 5.0(\square), 6.0(\diamond)$. Errors have been evaluated using bootstrap with 1000 samples.

Ref. [14], we set the physical scale by considering $3 d$ $S U(2)$ lattice results for the string tension and the input value $\sqrt{\sigma}=0.44 \mathrm{GeV}$, which is a typical value for this quantity in the $4 d S U(3)$ case. Since we consider $\hbar=c=1$, this implies $1 \mathrm{fm}^{-1}=0.4485 \sqrt{\sigma}$.

We find that the real-space propagator $C(t)$ is negative for several values of $t$, showing a clear oscillatory behavior (see Fig. 10. In analogy with Ref. 10] we also plot, in Fig. 2] the function $|C(t)|$ : the spikes reveal the change of sign in the propagator $C(t)$. Finally, in Fig. 3 we plot the function $-G(t, 1)$ : one can see that, as in the Gribov-like propagator, $G(t, 1)$ is negative for all values of $t$. Thus, we find an explicit violation of positivity for the lattice Landau gluon propagator. Let us stress that this violation is clearly observable for the three lattice couplings and for the two lattice volumes considered.

\section{SCALING AND FITS FOR $C(t)$}

It is important to check if the behavior obtained for $C(t)$ satisfies scaling for the lattice parameters considered here. To this end, we apply to the data the matching procedure described in [14, Section III] and consider $t$ in physical units using [14, Table 2]. We obtain that all propagators become negative at $t \approx 0.7 \mathrm{fm}$ and that the minimum is reached at $t_{\min } \approx 1 \mathrm{fm}$ (see Fig. (4). Moreover, finite-size effects seem to become important only at $t \gtrsim 3 \mathrm{fm}$. This means that our data for $t \in[0,3] \mathrm{fm}$ are essentially infinite-volume continuum results. Note that the Gribov-like propagator $C(t)$ in Eq. (10) has its minimum at $t_{\text {min }}=\pi /(M \sqrt{2})$. Thus, the above result for $t_{\min }$ would imply $M \approx \pi / \sqrt{2} \mathrm{fm}^{-1} \approx 2.22 \mathrm{fm}^{-1}=$ $438 \mathrm{MeV}=0.995 \sqrt{\sigma}$. Let us also observe that the momentum-space Gribov-like propagator $D(p)$ [see Eq. 


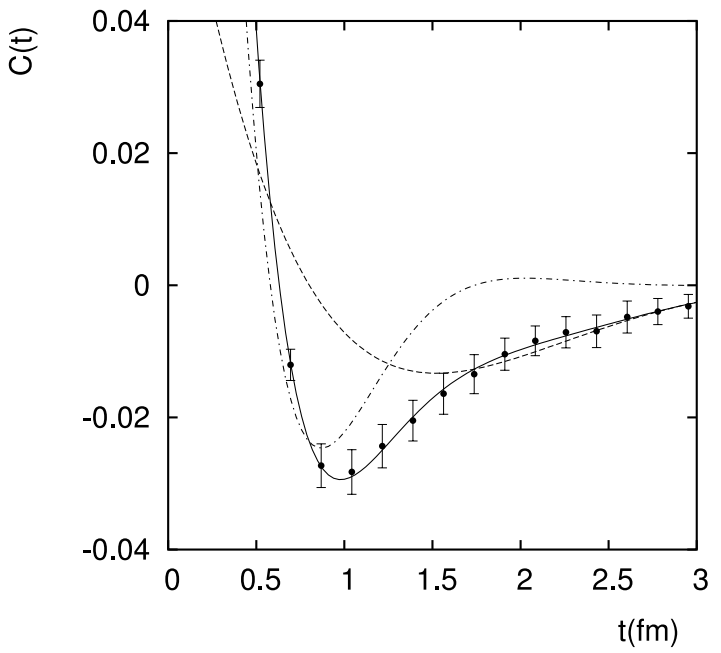

FIG. 5: Fit of $C(t)$ as a function of $t$ (in $f m$ ) using a sum of two functions of the type (17) for lattice volume $V=140^{3}$ and coupling $\beta=4.2$. We also display $C_{1}(t)$ and $C_{2}(t)$ separately.

(8)] has its maximum at $p_{\max }=M$. In Ref. 14] we obtained $p_{\max }=0.8_{-0.1}^{+0.2} \sqrt{\sigma}=350_{-50}^{+100} \mathrm{MeV}$.

As said above, in Ref. [14 we have fitted the gluon propagator in momentum space using a modified Gribovlike or Stingl-like formula with four or five parameters. We now try to fit the data in real space using

$$
C(t)=c e^{-\lambda t / \sqrt{2}} \cos (b+\lambda t / \sqrt{2}),
$$

which is a generalization of the Gribov-like propagator in Eq. (10). Clearly, this function also corresponds to a $G(t)$ that is always negative. Let us stress that only for $b=\pi / 4$ does this propagator correspond to the Gribov-like propagator in Eq. (8). In particular, for $b=0$ one gets the momentum-space propagator $D(p)=\left(p^{2}+M^{2}\right) /\left(p^{4}+M^{4}\right)$, which is finite at zero momentum. As reported in 14], it is still not clear from our data (on a $140^{3}$ lattice) if the zero-momentum gluon propagator vanishes in the infinite-volume limit, as predicted in [5, 6, 8$]$.

We fit the data obtained for the two largest physical volumes, i.e. $V=140^{3}$ and $\beta=4.2,5.0$, with $t$ in the range $[0,3] \mathrm{fm}$. As can be seen in Fig. 5 the data are well fitted using a sum of two functions of the type (17). The corresponding fitting parameters are reported in Table [ The averaged mass scales are $\lambda_{1}=1.69(1) \sqrt{\sigma}=$ $745(5) \mathrm{MeV}$ and $\lambda_{2}=0.74(1) \sqrt{\sigma}=325(6) \mathrm{MeV}$. One can also obtain good fits of the data in the whole $t$ range by considering the Fourier transform of the sum of three Stingl-like propagators in momentum space. These fits (using 12 parameters) have been reported elsewhere [21]. It is evident that fits of the gluon propagator in real space (see also [4]) require more parameters than fits in momentum space. This is due to the fact that the infrared data, for which the modeling is still not well understood, are spread over the whole time interval by the Fourier transform done in the evaluation of the temporal correlator $C(t)$.

Recently, it has been suggested 22, 23] that the violation of spectral positivity in lattice Landau gauge be related to the quenched auxiliary fields used for gauge fixing. We note that the fitting form proposed for $C(t)$ in [23] (also considering 5 fitting parameters) describes reasonably well our data up to $t=3 \mathrm{fm}$ - yielding a light-mass scale of about $1.14 \sqrt{\sigma}=500 \mathrm{MeV}$ - but cannot account for the oscillatory behavior observed at very large separations.

\begin{tabular}{cccccc}
$\beta$ & $c_{1}$ & $\lambda_{1}$ & $c_{2}$ & $b_{2}$ & $\lambda_{2}$ \\
\hline 4.2 & $0.368(6)$ & $3.83(4)$ & $0.70(3)$ & $0.099(6)$ & $1.54(4)$ \\
\hline 5.0 & $0.361(6)$ & $3.72(3)$ & $0.56(3)$ & $0.089(6)$ & $1.75(5)$ \\
\hline
\end{tabular}

TABLE I: Fit of the data using a sum of two functions of the type (17), setting $b_{1}=0$. We obtain $\chi /$ d.o.f. $\approx 0.24$ (respec. 0.19) for $\beta=4.2$ (respec. 5.0). The number of d.o.f. is 13 (respec. 17). The values of $\lambda_{1}$ and $\lambda_{2}$ are in $\mathrm{fm}^{-1}$. The relatively small $\chi /$ d.o.f. is probably due to the use of the diagonal part of the covariance matrix only.

\section{CONCLUSIONS}

Using data from the largest lattice sides to date, we verify explicitly (in the $3 d$ case) the violation of reflection positivity for the $S U(2)$ lattice Landau gluon propagator. This is one of the manifestations of confinement discussed in [1]. For very large separations $(t>3 \mathrm{fm})$ the propagator shows a clear oscillatory behavior, but of course one needs a careful extrapolation to infinite volume in order to verify if this behavior survives in that limit. In the scaling region, the data are well described by a sum of Gribov-like formulas, with a light-mass scale $M \approx 0.74 \sqrt{\sigma}=325 \mathrm{MeV}$, where $\sigma$ is the string tension. As a final comment, one should always bear in mind that the Gribov-like propagator may not represent the true analytic structure of the gluon propagator, but it is illustrative of a possible mechanism of confinement for the gluons (see also the discussion after Eq. (18b) in 24]).

\section{ACKNOWLEDGMENTS}

The authors thank Dan Zwanziger for helpful comments. Research supported by FAPESP (Projects No. 00/05047-5 and 03/05259-0). Partial support (AC, TM) from $\mathrm{CNPq}$ is also acknowledged.
[1] R. Alkofer and L. von Smekal, Phys. Rept. 353, 281 (2001).
[2] Local Quantum Physics, R. Haag (Springer, Berlin, 2nd edition, 1996). 
[3] K. Osterwalder and R. Schrader, Commun. Math. Phys. 31, 83 (1973); Commun. Math. Phys. 42, 281 (1975); Statistical Field Theory, G. Parisi (Addison-Wesley, Redwood City, 1988).

[4] H. Aiso et al., Nucl. Phys. Proc. Suppl. 53, 570 (1997).

[5] D. Zwanziger, Nucl. Phys. B 364, 127 (1991).

[6] D. Zwanziger, Nucl. Phys. B 412, 657 (1994).

[7] V. N. Gribov, Nucl. Phys. B 139, 1 (1978).

[8] D. Zwanziger, Phys. Lett. B 257, 168 (1991).

[9] L. von Smekal, A. Hauck and R. Alkofer, Phys. Rev. Lett. 79, 3591 (1997); J. C. R. Bloch, Phys. Rev. D 64, 116011 (2001); D. Zwanziger, Phys. Rev. D 65, 094039 (2002); C. Lerche and L. von Smekal, Phys. Rev. D 65, 125006 (2002); C. S. Fischer, R. Alkofer and H. Reinhardt, Phys. Rev. D 65, 094008 (2002); D. Zwanziger, Phys. Rev. D 67, 105001 (2003).

[10] R. Alkofer, W. Detmold, C. S. Fischer and P. Maris, Phys. Rev. D 70, 014014 (2004).

[11] A. Cucchieri, Nucl. Phys. B 508, 353 (1997); Phys. Lett. B 422, 233 (1998); Phys. Rev. D 60, 034508 (1999); H. Nakajima and S. Furui, Nucl. Phys. Proc. Suppl. 73, 635 (1999). F. D. Bonnet et al., Phys. Rev. D 64, 034501 (2001); I. L. Bogolubsky and V. K. Mitrjushkin, arXiv:hep-lat/0204006

[12] A. Cucchieri, F. Karsch and P. Petreczky, Phys. Lett. B
497, 80 (2001); Nucl. Phys. Proc. Suppl. 94, 385 (2001).

[13] A. Cucchieri, F. Karsch and P. Petreczky, Phys. Rev. D 64, 036001 (2001).

[14] A. Cucchieri, T. Mendes and A. R. Taurines, Phys. Rev. D 67, 091502(R) (2003).

[15] S. Furui and H. Nakajima, hep-lat/0403021

[16] A. Cucchieri and D. Zwanziger, Phys. Rev. D 65, 014001 (2002); Phys. Lett. B 524, 123 (2002).

[17] D. B. Leinweber et al., Phys. Rev. D 60, 094507 (1999) [Erratum-ibid. D 61, 079901 (2000)].

[18] J. E. Mandula, Phys. Rept. 315, 273 (1999).

[19] Quantum Fields on a Lattice, I. Montvay and G. Munster (Cambridge University Press, Cambridge, 1994).

[20] J. E. Mandula and M. Ogilvie, Phys. Lett. B 185, 127 (1987); C. W. Bernard, C. Parrinello and A. Soni, Phys. Rev. D 49, 1585 (1994).

[21] A. R. Taurines, A. Cucchieri and T. Mendes, hep-lat/0408022

[22] C. A. Aubin and M. C. Ogilvie, Phys. Lett. B 570, 59 (2003);

[23] C. Aubin and M. C. Ogilvie, Phys. Rev. D 70, 074514 (2004).

[24] Y. L. Dokshitzer and D. E. Kharzeev, hep-ph/0404216 